\title{
THE PRESENT DAY TREATMENT OF TUMOURS OF THE PROSTATE.
}

By CLIFFORD MORSON, O.B.E., F.R.C.S.

(Surgeon, St. Peter's Hospital for Stone and Hampstead General Hospital.)

The prostate gland is an organ which is susceptible to attack by many diseases, partly on account of its structure but particularly because of its anatomical relationship to the urinary conduit.

\section{ADENOMATOSIS.}

One of its most common affections is adenomatosis, or as it is popularly termed, simple enlargement. The assertion is often made that the large majority of elderly men suffer from this disease, but the supposition is based. more on apprehension than on accurate observations.

Many of those afflicted require no more treatment than a careful mode of living, for frequency of micturition per se is not an indication for either operation or instrumental interference. On the other hand, a change in the normal intervals of passing urine must never be regarded as evidence merely of the onset of senility. If the prostate is responsible for the frequency, the change in the gland is not that of the physiological process of old age, but of a definite pathological lesion.

The symptoms of adenomatosis or simple enlargement depend upon the changes in the prostatic urethra and the bladder. As the tumours develop so is the anatomy of the surrounding parts altered. The adenomata often bulge into the lumen of the urethra so that it takes on an $S$ shaped curve or they may grow into the bladder and cause the internal meatus to look directly forwards, thus making catheterisation very difficult. These are extreme degrees of urethral deformity. In the early stages the alteration from the normal is obviously slight and the changes can only be recognized by the expert cysto-urethroscopist. The verumontamum is lengthened or even flattened out and the internal meatus reveals the projection of one or more tiny adenomata.

There is no need to discuss in detail the effect of prostatic obstruction upon the bladder musculature. These effects are too well known to the post-graduate student to require more than a passing reference. However, it is important that he should fully understand the mechanism which leads to the formation of residual urine and the power of recovery of an atonic bladder. Successful treatment is dependent upon a correct appreciation of the causes which operate to produce the main symptoms of this disease. Residual urine is only formed when the internal meatus is raised above the level of the bladder base. The growth of adenomata behind the vesical end of the prostatic urethra tends to push the internal meatus towards the pubis. At the same time there may be developing the so called middle lobe which, if it reaches sufficient size overhangs the trigone. This middle lobe is nothing more that a pedunculated mass of adenomata which has spread upwards into the bladder cavity. Both these factors determine the size of the post-prostatic pouch, and therefore the amount of residual urine. Deterioration of the vesical musculature is an additional factor.

Adenomatosis per se is not a disease which kills, and recourse to a radical cure, which for lack of knowledge of the cause means a surgical procedure, is only indicated when the secondary changes are threatening life. There are many cases where the symptoms are just a nuisance and no more. With such patients any treatment which is attended by risk to life is absolutely unwarrantable. 
Two investigations are necessary before the urologist is entitled to give a considered opinion concerning the line of treatment to be adopted. Firstly, he must measure the amount of residual urine, and secondly, determine the degree of damage to the renal function, if any.

The amount of residual urine is a good working guide to the progress of the disease, and can only be accurately estimated by catheterization immediately after micturition.

The exception is when a large diverticulum is present. Under such conditions the total quantity of retained urine can only be measured with the aid of the catheterizing cystoscope.

The test of renal function requires a much more complicated investigation and demands above all things a sound clinical knowledge of the manifestations which the smallest degree of failure may produce upon other organs. The vascular system and digestive organs are quickly affected by urinary back pressure.

The well known laboratory tests, such as the estimations of urea in the blood and in the urine are never to be relied on as infallible guides to renal function.

The presence of sepsis must also not be ignored where considerations of treatment are concerned, but patients with long standing urinary infection are often better surgical risks than those who have sterile urines.

If a man has suffered from sepsis over a long period he has acquired an immunity, with which the clean case has not been favoured. The degree of immunity is best determined by a study of the general rather than the local condition. In the early days of prostatectomy every case submitted to operation suffered from cystitis and yet Freyer's mortality rate figures have compared favourably with those of any surgeon except Harry Harris.

Having considered briefly the steps which must be taken to enable the urologist to give a reliable opinion on treatment the way is open to discuss the various methods which are available to relieve or cure the prostatic.

What advice are we to give to the patient in the early stages of adenomatosis?

Frequency is confined to once or at most twice nightly and the residual urine is never more than one ounce. There is no evidence that the kidneys are other than healthy. The patient is anxious because he knows he has reached the prostatic age and micturition is not quite normal.

A very large number of such cases remain in statu quo, the adenomata for some unknown reason making no further growth.

It is wise to allay the patient's fears by informing him of these facts, but to warn him that indiscretions of diet, and mode of life may bring on an attack of retention. He should be instructed as a matter of precaution to get his residual urine tested at intervals of twelve months.

We are profoundly ignorant how an adenoma's growth can be arrested, and when, in the early stages of the disease, retention occurs, it is due to congestion of the mucous membrane of the internal meatus and prostatic urethra and not to the size of the tumour. The two common causes of retention in these cases are either a bacillus coli infection, or a sudden and rapid diuresis.

Colon bacilluria is as frequently observed in an otherwise healthy urinary tract as it is in association with some persisting disease and therefore one or more small adenomata growing in the prostate cannot be necessarily accused of initiating this complication. 
The surgeon who urges removal of the tumours under these conditions is risking his own reputation as well as the life of his patient, for should the latter refuse operation after the attack of retention has passed, he will say hard things about the surgeon's enthusiasm for knife or cautery. Treatment in these cases consists of passing into the bladder through the urethra a Malacot tube No. I8F size, with two eyes. This catheter does not have to be tied to the penis and is better tolerated than any other variety. This instrument should be removed at the end of a week, when micturition, apart from frequency will have been restored to normal. While the catheter is in situ bladder lavage is contra indicated. Its adoption increases the inflammation and therefore pain. The tube can be kept clean by the patient's diuresis, which is maintained by copious drinks supplemented if necessary by rectal injections of water. The infection can be further controlled by the usual anti bacillus coli methods.

It has to be admitted that cure of the colon bacilluria cannot be guaranteed, and therefore retention may recur should there be a further attack of acute cystitis. This, however is extremely rare for the infection tends to become chronic.

The other cause of failure of micturition, namely a sudden and rapid diuresis, is the outcome of dietetic error or mode of life. The tendency of an affluent elderly man to overdrink and overeat is well known, and, when investigation has shown that the mucous membrane covering a prostatic adenoma readily swells up, it requires but little imagination to visualize the effect of his indiscretions upon the act of micturition.

One of the common causes of sudden diuresis is a rapid fall in the atmospheric temperature. This is well illustrated when a man leaves a warm room for the cold air of the street, as he will do after enjoying the hospitality of a friend at dinner.

All patients with early adenomatous disease of the prostate must be instructed to micturate immediately before leaving the artificially heated atmosphere of a house for the normal temperature of the external air. Retention is a frequent sequel to a good dinner with alcohol, followed by neglect to empty the bladder before motoring home.

Again, to ignore the urge of micturition is often sufficient to involve the early prostatic in catheterization. Thus it will be seen that a careful attention to the physiological processes of the urinary organs and avoidance of those excesses which increase their work are sufficient to prevent the patient with slight adenomatous disease from requiring any local treatment.

The next type of case to be considered is the one which on repeated examination over a period of months or years shows a steady increase in the amount of residual urine. A three ounce retention is putting the patient in the danger zone of renal impairment and therefore local treatment is indicated.

To-day there are two schools of thought, each of which claims that its views on treatment are correct. The one is sponsored and powerfully supported by the majority of urologists in America. These investigators believe that transurethral resection of portions of the enlarged prostate by the electric current will enable the patient to avoid what they consider to be the formidable operation of prostatectomy. The other, which includes among its adherents the best known surgeons in Europe, advocates an operation which aims at producing a radical cure.

Treatment by the electric current was introduced by Bottini in 1885 , but since that date it has undergone many changes as the result of the invention of various 
kinds of instruments. Some of these punch pieces out of the adenomata while others cut.

The object of this surgical procedure is to make a gutter in the region of the posterior lip of the internal meatus and shave off pieces of the lateral projections of adenomatous tissue. This operation may be termed closed partial prostatectomy as opposed to the open, which consists of a cystotomy with removal of the intra-vesical portion of the enlarged gland. This latter has been proved a failure. The evidence in favour of the former is conflicting.

It will be agreed by most urologists that except in the largest adenomatous prostates temporary improvement in symptoms is likely to follow the use of this method, but the disease is far from cured and the anatomical changes in the bladder remain. Moreover, the danger from such complications as sepsis and hæmorrhage is as great as in complete prostatectomy by the modern technique.

It may be argued that as there are some cases in which there is spontaneous arrest of growth of the adenomata, the shaving off of parts of the tumours will cure the patient of his symptoms. Unfortunately, we know that in the analagous case of partial tonsillectomy complete removal has to be subsequently performed. Likewise it is the experience of the writer that patients who submitted to transurethral resection three years ago are now returning with a recurrence of symptoms.

What then are the advantages of this limited operation over complete prostatectomy and in what type of patient is it indicated?

Firstly, there is relatively little shock and secondly, confinement to bed is usually limited to a fortnight. More than one advocate of this mode of treatment has erroneously emphasized certain economic advantages, particularly in the saving of surgical dressings.

It is doubtful whether by the Harris technique for prostatectomy the average cost of dressings per patient works out at more than half-a-crown. Whichever type of resection instrument is used the results are partly dependent upon the skill of the operator. This is equally true of complete prostatectomy. Statistics are notoriously fallacious.

The mortality rate of prostatectomy may be as low as 2.7 per cent. and as high as I4.2 per cent.; of transurethral resection from I to II per cent. Such a wide range in both operations suggests serious errors of technique.

In the opinion of the writer there are few cases of adenomatous disease of the prostate which should be submitted to transurethral resection.

As has already been emphasized the man with a small quantity of residual urine requires no local treatment. Transurethral resection will expose this type of case to the dangers of infection.

If the local disease is complicated by cardiac changes, it is wise to determine the cause of the heart lesion before deciding on treatment of the prostate.

So many of these patients are suffering from a toxæmia that drainage of the bladder by supra-pubic cystotomy is just as imperative for the sake of the heart as it is for the kidneys. On the other hand, should it be clearly demonstrated that the vascular lesion is independent of the prostatic obstruction, then common sense dictates that any surgical procedure attended by shock will lead to disaster.

These are the cases which can be submitted to transurethral resection. 
The operations which are practised today for the radical cure of the adenomatous prostate are by two routes, the perineal and the supra-pubic. The former has never found much favour in this country, for the technique is more difficult for the average surgeon, and may be attended by such complications as a recto-vesical fistula and incontinence of urine.

The operation by the supra-pubic route, according to the Freyer technique, can be accomplished by most surgeons with fair success, but if the results are to be improved then the Harry Harris technique with the modifications which the writer has introduced must be mastered.

Harris has shown that the principles which surgeons have adopted in other parts of the body can be applied with success to the prostatic region.

Put quite simply it means that after removal of the diseased tissue of the prostate reconstruction of the anatomical structures is possible.

The young urologist cannot expect an easy path to success in modern prostatectomy. He has not only to possess sound judgment in pre and post operative treatment, but he requires nimble fingers to master the special instruments.

It is absolutely necessary that he should see what he is doing within the bladder and therefore all illumination must be concentrated in the operation area.

All retractors for this operation now have electric light attachments. There are three lamps for the bladder and one for the prostatic cavity.

With the aid of the latter and Morson's prostatic speculum it is possible to observe all the tissues which form its walls. The fractured end of the urethra is thus visible, as well as the prostatic bed. Every attempt must be made under direct vision to approximate the mucous membrane of the trigone to that of the urethra by stitches. The next step is to obliterate the prostatic cavity after insertion of the urethral catheter, and control hæmorrhage. This is accomplished by a figure-of-eight stitch which passes through the lateral walls. The post-graduate student can only learn the details of the technique by watching the operation performed by a urologist who is thoroughly conversant with its various details. A written description affords little idea of this latest surgical procedure.

Unfortunately, though success in hæmostasis has been attained, we are a long way yet from obtaining control of either pre or post operative sepsis.

The prostatic with a chronic colon bacilluria cannot be cured of his infection by either resection or the radical operation. Even prolonged drainage is of no avail, the improvement in health with a cystotomy being due, not to reduction in sepsis, but the mechanical effect on the kidney of relief from back pressure.

Urethral instrumentation before operation should be avoided wherever possible on account of the danger of infecting both prostate and vesiculæ seminales. A catheter retained in the urethra for more than forty-eight hours will, without exception, cause infection of the adenomatous mass, and this may be accompanied by vesiculitis. It is for this reason that attempts should be made to sterilize these organs before commencing the operation. It is my practise to irrigate the vesiculæ seminales with one in sixty carbolic acid immediately prior to enucleation of the prostatic adenomata. This technique is simple. The vas is divided at the external abdominal ring. This manouvre prevents infection which may be present in the bed of the prostate after operation from travelling to the testicle. Into the proximal end of the cut vas is injected with a needle and syringe Io to 20 c.c. of the antiseptic fluid. The latter distends both seminal vesicle and prostatic urethra. (See Fig. I6). It is often recommended that a urinary antiseptic such as hexamine be given by mouth prior to the operation, but no drug 
has yet been discovered which will destroy the organisms in a chronically infected kidney without serious damage to the cells of the latter. The problem, therefore, of how to sterilize the urinary conduit still remains to be solved. Once this has been solved the operation of prostatectomy will be attended by no more risk than that of the removal of a lipoma.

Before leaving the subject of the treatment of adenomatosis it is necessary to refer to the methods which must be adopted to prepare for operation the patient who suffers from chronic over distension of the bladder. This is an extremely serious condition which demands the utmost patience from the doctor and patient alike. The ureters and kidneys are permanently damaged by the prolonged back pressure, but life is possible with so little healthy kidney tissue that often in the worst cases hope need not be abandoned of saving the patient.

First and foremost the bladder must be emptied so slowly that at least forty eight hours are required to accomplish it. Hæmorrhage from the bladder mucous membrane and even uræmia are accompaniments of rapid evacuation of urine. The apparatus needed for this treatment is quite simple. Whether the drainage be by indwelling catheter or supra-pubic tube a glass dripper is attached to the end of it, the lumen of which should only admit a fine bristle. This is used when the urine is clear. If it is full of mucus and pus, a clip must be fixed to the rubber tube and a nurse instructed to allow one or two ounces to escape every quarter of an hour.

It is the exception to find the patient with chronic overdistension fit for prostatectomy after decompression has been accomplished. More likely it is that prolonged drainage is needed and therefore the indications are for immediate supra-pubic cystotomy rather than the indwelling catheter. A rise of temperature usually follows the emptying of the bladder, the tongue is dry and anorexia is present. As soon as the stage of reaction has passed steps must be taken to determine the amount of destruction of renal tissue. Intravenous pyelography gives more accurate information in this respect than any laboratory test. If the injected fluid is not excreted by either kidney, the damage done by back pressure is irretrievable and in permanent supra-pubic cystotomy lies the only hope of prolongation of life. Should the calices fill well, but the pelvis is shown to be dilated, prostatectomy after a period of drainage can be performed with success. Sometimes only one kidney appears normal, the other failing to excrete or being markedly hydronephrotic. Again after drainage a good prognosis from prostatectomy may be given. Slow excretion by the kidneys is not a bar to the major operation provided that the X-ray shadow shows well formed calices. In some cases none is observed until 30 minutes have elapsed after the injection.

Because of the value of intravenous pyelography the post-graduate student must not assume that the blood urea and urea concentration tests are not needed. Every piece of information that may assist in the accurate assessment of renal function is urgently required in such serious cases. (See paper by Dr. Cuthbert Dukes, p. 390).

Before finally leaving the subject of adenomatosis of the prostate, one other complication must be mentioned. These tumours have a blood supply in proportion to their size, and if the patient has arterio-sclerosis, rupture of one of the arteries feeding the growth is by no means uncommon. This hæmorrhage may be so severe as to cause clot retention or sufficiently slight merely to tinge the urine. However moderate in amount, bleeding is a danger signal of worse to come, if not radically treated, and therefore irrespective of the quantity of residual urine, prostatectomy is indicated. 


\section{OTHER TUMOURS OF PROSTATE.}

There are three other varieties of tumour formation besides the adenoma which grow in the prostate gland. They are the myoma, the sarcoma and the carcinoma. The two former are rare, the latter unfortunately not so.

The myoma cannot be distinguished in the clinical state from the adenoma, and at operation is as easily enucleated. The correct diagnosis is revealed by the pathologist. The sarcoma, on the other hand, is an infiltrating growth and though the major portion of it may be enucleated its local recurrence is almost a certainty. The writer has never seen a case cured no matter what treatment has been adopted. Ablation by surgical means is inadvisable, the best procedure being supra-pubic cystotomy followed by irradiation. The condition occurs in infants and children, rarely young adults, and does not often come under medical observation until retention has occurred.

There are two types of carcinoma. from an adenoma.

The scirrhus and that which develops

The scirrhus type is uniformly hard and not difficult to diagnose by rectal examination. The adeno-carcinoma on the other hand, often by clinical examination, deceives the investigator. In the early stages mobility of the prostate gland is unaffected and infiltration of the surrounding tissues absent. A hard nodule in an otherwise elastic prostate may be just as likely an adenoma as a carcinoma. Even the diagnosis of calculus must not be ruled out in the absence of an X-ray picture. It should be a golden rule therefore, when in doubt, to insist on suprapubic cystotomy. Transurethral resection for the purposes of diagnosis cannot be relied on, the pieces removed by this operation consisting so often of thickened mucous membrane with an entire absence of tumour tissue.

Should the cystotomy reveal a carcinoma, the writer's inclination after trial, of every known method of treating cancer during the past twenty-five years, is to give the patient a permanent supra-pubic apparatus.

Radium, X-rays and diathermy, singly or in combination, have been extensively tried out. In many cases improvement in micturition follows, but eventually the patient has to submit to permanent supra-pubic cystotomy. The writer, some years ago, introduced a radical operation which consisted of the removal not only of the prostate but also the seminal vesicles and base of bladder. Disappointment in the results has led to its discontinuance. No patient survived longer than five years.

Is the surgeon justified in performing a severe operation when the expectation of life is limited to that period? We think not, since the anxiety of threatened retention can be avoided by a minor operation.

Diathermy treatment is equally disappointing. The growth has been attacked by either the open or closed technique and again recourse finally has had to be made to supra-pubic cystotomy.

It is true that most urologists have had experience of removing an adenomatous mass in which was a small carcinoma, and the patient has survived the operation many years. The surgeon who treats internal cancer from time to time has such an experience, but let him not boast of successes which so rarely come to hand. Those who have been in surgical practice for over twenty-five years, if they be true to themselves where the results of cancer treatment are concerned, are the most modest of men. 